\title{
Towards Automated Extraction of EMC-Aware Trajectory-Based Macromodels for Analog Circuits
}

\author{
Georges Gielen \\ ESAT-MICAS \\ K.U. Leuven \\ Heverlee, Belgium 3001 \\ Email: gielen@esat.kuleuven.be
}

\author{
(Invited Paper) \\ Dimitri De Jonghe \\ ESAT-MICAS \\ K.U. Leuven \\ Heverlee, Belgium 3001 \\ Email: ddejongh@esat.kuleuven.be
}

\author{
Johan Loeckx \\ ESAT-MICAS \\ K.U. Leuven \\ Heverlee, Belgium 3001 \\ Email: jloeckx@esat.kuleuven.be
}

\begin{abstract}
In this paper a new methodology is proposed for automated generation of EMC-aware behavioral models. Tightening EMC regulations require elaborate testing and simulation of EMC susceptibility of analog circuits during the design stage. A classification strategy detects EMC-sensitive devices which allows us to incorporate a more elaborate device model when accuracy is needed due to rectification effects. In addition, trajectory-based models are used for efficient modeling of nonlinear dynamical systems. Trajectory methods sample the state space trajectory of a circuit while exciting the circuit with a representative training input. The macromodel is generated by reduction and interpolation of linearizations among the sampled trajectory points, in which the validity of the model is defined. Without relevant loss of accuracy, the simulation time of larger systems for EMC can be reduced with an order of magnitude. The method is verified on a single-stage opamp with rectifying bias current mirror, a circuit exhibiting EMC distortion.
\end{abstract}

\section{INTRODUCTION}

The complexity of modern mixed-signal integrated systems in automotive electronics increases with the number of electronic systems assembled in these cars. Degradation of electromagnetic compatibility (EMC) quality also scales with the number of digital integrated circuits, controlled sensors and actuators. Tightening EMC regulations challenge CAD to develop simulation techniques that are less time consuming without significant loss of accuracy. Macro- or behavioral models are simplified representations of the original circuit, speeding up the simulation time while retaining the accuracy needed to model the complexity of real analog systems.

The most predominant approach towards macromodeling of mixed-signal nonlinear systems is manual abstraction. Recent research developments however enable an on-demand automated extraction of behavioral models [1]. Whitebox modeling techniques like model-order reduction (MOR) reduce the state space equations of a linear system by direct moment matching methods [2] [3]. A linear time-invariant system with state vector $\mathbf{x}(t)$ and input vector $\mathbf{u}(t)$ can be written as a set of differential equations

$$
\left\{\begin{array}{l}
E \frac{d \mathbf{x}(t)}{d t}=A \mathbf{x}(t)+B \mathbf{u}(t) \\
\mathbf{y}(t)=C^{T} \mathbf{x}(t)+D \mathbf{u}(t)
\end{array}\right.
$$

The size of $\mathbf{x}(t)$ might be quite large but can be reduced by varying model-order reduction techniques such as Asymptotic Waveform Evaluation (AWE) [4], Krylov subspace projection [5] and Truncated Balanced Reduction (TBR) [6].

As most mixed-signal blocks are non-linear devices, modelorder techniques have been extended to handle weakly nonlinear systems. Rewienski and White proposed a piecewise approximation technique, 'Trajectory Piecewise Linear' (TPWL), that is also applicable to some strongly nonlinear systems [7]. This method has successfully been extended with scaling capabilities [8] and higher-order approximations [9].

In the presence of Electromagnetic Interference (EMI), the nonlinear behavior of devices that normally operate in a linear fashion becomes important [10]. Since this disturbance spans a large range of frequencies and amplitudes, the state space to be characterized tend to explode [8]. Also, the calculation of a stationary solution requires a long and computationally expensive transient simulation. For this reason, a complete exploration during model building is not feasible.

The problem we address in this paper is how to generate on-demand EMC-aware behavioral models. Our approach generalizes the trajectory-based modeling algorithm, allowing other circuit representations than a set of differential equations to be implemented. We also foresee a flexible integration of multiple layers of abstraction to improve the accuracy of relevant devices.

This paper is organized as follows. Section 2 provides the necessary background of the trajectory method, designates some problems and proposes a generalization of the trajectory methodology. Section 3 describes a practical implementation for a generalized trajectory methodology. Section 4 evaluates the proposed algorithm and section 5 concludes.

\section{TRAJECTORY BASED Macromodels}

Trajectory-based methods focus on strongly nonlinear dynamical systems, represented by a set of first-order differential equations in their state space form:

$$
\left\{\begin{array}{c}
\frac{d g(\mathbf{x}(t))}{d t}=f(\mathbf{x}(t))+B(\mathbf{x}(t)) \mathbf{u}(t) \\
\mathbf{y}(t)=C^{T} \mathbf{x}(t)
\end{array}\right.
$$


where $\mathbf{x}(t) \in R^{N}$ is a state vector evolving with time for a given system, $f(),. g():. R^{N} \rightarrow R^{N}$ are nonlinear vectorvalued functions, $B($.$) is a state-dependent (but often taken as$ a constant) $N \times M$ input matrix, $\mathbf{u}(t) \in R^{M}$ is an input to the system and $C$ is a $N \times K$ mapping to the output vector $\mathbf{y}(t) \in R^{K}$.

\section{A. Background}

In TPWL a large amount of center points (samples) is selected along a simulation trajectory in the state space as a representative training input excites the circuit. Each sample is approximated by a linearization and further reduced with MOR techniques. Different piecewise regions are combined by weighting functions, generating a combined linear model that covers most nonlinearities [7]:

$$
\begin{aligned}
& \frac{d}{d t}\left[\sum_{i=0}^{n-1} w_{i}(\mathbf{x}(t))\left(g\left(\mathbf{x}_{\mathbf{i}}\right)+G_{i}\left(\mathbf{x}(t)-\mathbf{x}_{\mathbf{i}}\right)\right)\right] \\
& =\sum_{i=0}^{n-1} w_{i}(\mathbf{x}(t))\left(f\left(\mathbf{x}_{\mathbf{i}}\right)+A_{i}\left(\mathbf{x}(t)-\mathbf{x}_{\mathbf{i}}\right)+B_{i}(\mathbf{x}(t)) \mathbf{u}(t)\right)
\end{aligned}
$$

However, the capability in representing weak nonlinearities within each region is limited since any higher-order nonlinearities are due to the smoothing functions $w_{i}(\mathbf{x}(t))$. In [9] a higher-order Taylor expansion, piecewise polynomial (PWP), is proposed. A quadratic expansion of $f(\mathbf{x}(t))$ in point $i$ is

$$
f\left(\mathbf{x}_{\mathbf{i}}\right)+A_{i}^{(1)}\left(\mathbf{x}(t)-\mathbf{x}_{\mathbf{i}}\right)+A_{i}^{(2)}\left(\mathbf{x}(t)-\mathbf{x}_{\mathbf{i}}\right) \otimes\left(\mathbf{x}(t)-\mathbf{x}_{\mathbf{i}}\right)
$$

where $A_{i}^{(1)}$ and $A_{i}^{(2)}$ are the first- and second-order derivatives and $\otimes$ is the Kronecker (tensor) product. This representation allows us to perform a small-signal distortion analysis instead of solely a large-signal transient analysis. However the amount of state space variables increases significantly due to the order of the tensor product.

To avoid an explosion of redundant samples in the state space, Tiwary and Rutenbar developed a scalable TPWL method with clustering [8]. This methodology prunes away redundant trajectory samples, gives a full BSIM3 support and enables an incremental model update. All these enhancements have made trajectory methods a very appealing and stable platform for widespread use.

\section{B. Modified Trajectory Method}

All trajectory methods discussed above exploit the mathematical state space representation that can easily incorporate algebraic operators to reduce the model order. This formulation of the system defines an input-to-output behavior by means of a set of first-order differential equations. A more flexible approach would allow us to model the nonlinear function from each terminal to another one. Suppose a spurious signal is injected in an output terminal or supply and causes operatingpoint changes in an internal node of the system (e.g. DC-shift due to rectification effects). This effect could fall beyond the scope of the traditional state space description because the output-to-input path is not necessarily included.

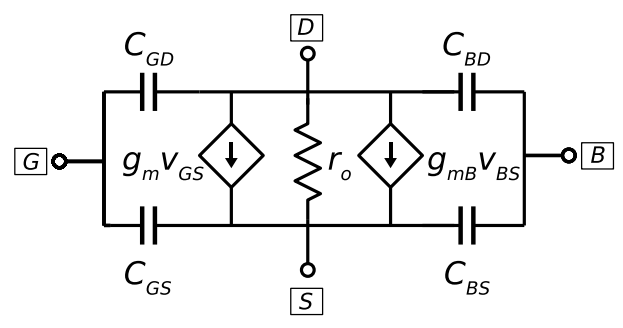

Fig. 1. Small signal macromodel for a BSIM3 transistor.

The trajectory method holds when another local model is applied instead of the linearized set of differential equations, as long as the model is valid in a range around the sample point. In this paper we use a small-signal network to capture the essential dynamics of a BSIM3 model, as shown in Fig.1. The validity of the model is guaranteed when the electrical parameters for each sample are extracted from the original DC-operating-point simulation in that sample.

According to the original trajectory method, the network parameters of different samples are combined by a weighting function $w_{i}(x(t))$ to generate a large-signal model. This implies that output resistance, for example, becomes a weighted sum of the extracted values for each sample, according to:

$$
r_{o}(\mathbf{x}(t))=\sum_{i=0}^{n-1} w_{i}(\mathbf{x}(t)) r_{o i}
$$

where $r_{o i}$ is the output resistance for the i-th sample.

\section{Layered Abstraction Modeling}

The above modified trajectory method allows a flexible definition of the models to be simplified, which does not necessarily needs to be a state space description. The level of abstraction can be localized and differ from device to device. If accuracy is wanted, highly nonlinear devices are modeled with a low abstraction level implementation, like a BSIM3 model for a transistor, at the cost of simulation speed. The range of a low abstraction level device is larger than a highly linearized device, preventing a local state space explosion. The choice of abstraction level can be determined by a sensitivity analysis of each device.

Low abstraction levels are for example a set of first-order differential equations, artificial neural networks, simplified macromodels, Norton equivalent circuits or another functional description of a nonlinear device. A smooth connection between the different model implementations is mandatory and can be realized with a co-simulation of a descriptive language like VHDL-AMS or Verilog-A and SPECTRE or SPICE.

\section{STEAM METHOdOLOGY}

Based upon the modified trajectory method and layered abstraction modeling discussed above, a Sensitivity-based Trajectory EMC-Aware Macromodeling strategy (STEAM) has been designed. The purpose of STEAM is to incorporate 
EMC-related effects in an on-demand behavioral model generation strategy. The generation and evaluation of this trajectory model is now further discussed in detail.

\section{A. Model Generation}

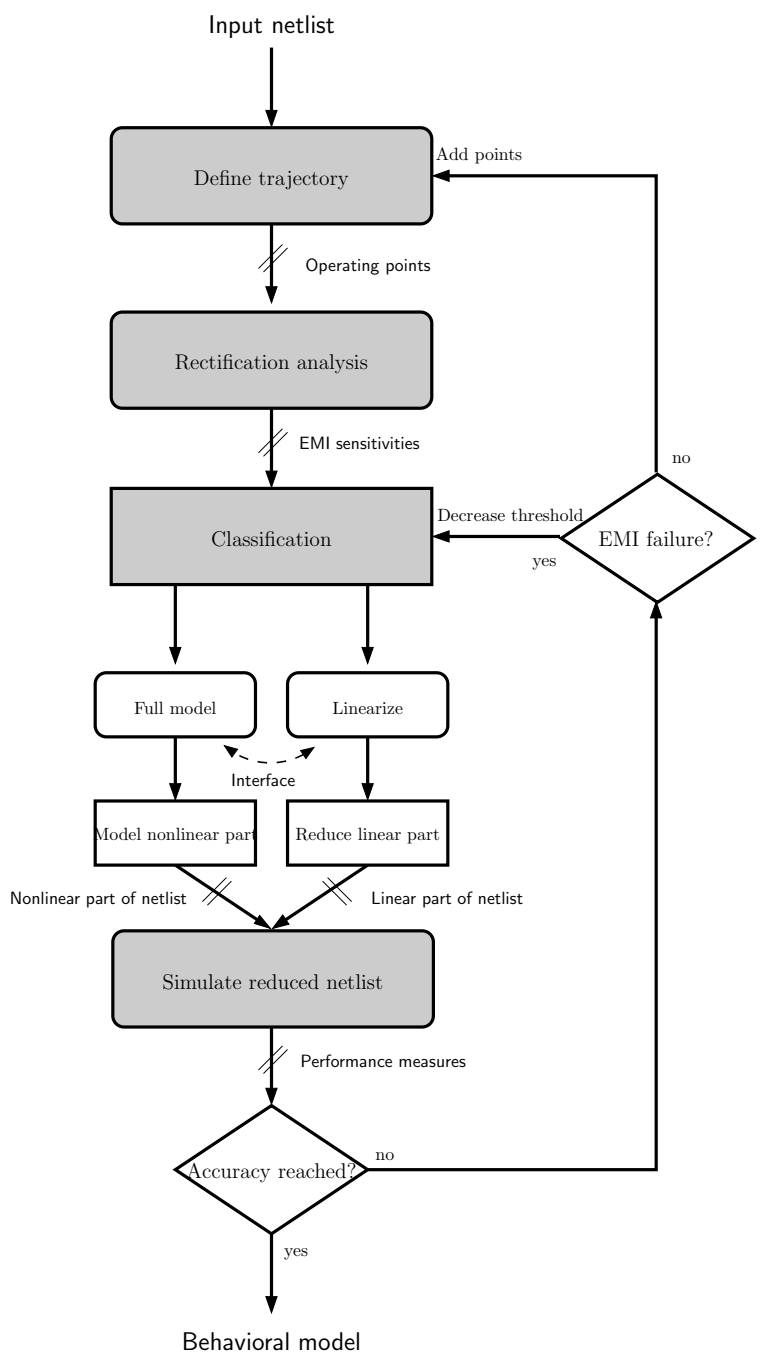

Fig. 2. STEAM model generation flow.

Fig.2 gives the model generation flow for the STEAM algorithm. The input is a circuit netlist in SPICE format and its corresponding operating range to define the state space boundaries. Some initial boundary samples of the trajectory are generated with a small perturbation analysis in different DCoperating-points along the trajectory. The electrical parameters of the circuit are then extracted from the analysis in each sample. Next, a rectification analysis in each sample predicts the EMI susceptibility of each device [11].

Each device is then classified as EMI-soft or EMI-hard. Devices that are labeled EMI-soft are implemented by their full-model implementation to maintain accuracy. EMI-hard devices are insensitive to EMI-induced rectification effects and are linearized. This linearization is based upon the macromodel of Fig.1 and its corresponding parameter extraction for each sample. The current model only implements two levels of abstraction (full BSIM3 and the macromodel) but can easily be extended to more layers. The full model is implemented in SPICE and the macromodels are written in VHDL-AMS. To interface the different levels of abstraction, the behavioral model only needs to be trained with the expected DCoperating-point changes of the full model devices.

To verify the accuracy of the generated model, the error between the trajectory-based model and the original circuit is measured and samples are added where the model was not validated. The threshold of our classification strategy is increased when EMI effects have not fully been modeled.

\section{B. Model Simulation}

During simulation the electrical parameters of the macromodels are evaluated using a weighted representation of the parameters. Because of the critical importance of a smooth transition between sampled regions, the weighting function is highly skewed in favor of the samples $\mathbf{x}_{\mathbf{i}}$ that are close to the current state $\mathbf{x}(t)$ [9]. The function is further normalized so

$$
\text { that } \begin{aligned}
\sum_{i}^{n-1} w_{i}(\mathbf{x}(t))=1: & \\
w_{i}(\mathbf{x}(t))= & \frac{\exp ^{-\left(S \delta_{i}\right)}}{\sum_{i}^{n-1} \exp ^{-\left(S \delta_{i}\right)}} \\
\delta_{i} & =\sum_{k}^{p-1}\left(\frac{x_{k}(t)-x_{k, i}}{x_{\max , k}-x_{\min , k}}\right)^{2}
\end{aligned}
$$

where $\delta_{i}$ is the relative distance of a sample to the current state for each state space dimension $k$. Parameter $S$ sets the skewness of the weighting function: the higher the value the more the skewness. $n$ is the number of samples and $p$ is the size of state vector $\mathbf{x}(t)$.

\section{EXPERIMENTAL RESULTS}

The STEAM algorithm has been implemented in Python for parameter extraction of SPECTRE and SPICE netlists. The behavioral model is co-simulated in VHDL-AMS and SPICE. Because of the high evaluation effort of the weighting functions, they are calculated up-front and implemented by means of a look-up table.

A model is extracted for a single-stage opamp, biased by a current mirror with a low-pass filter, see Fig.3. A 4mA EMI signal (100MHz sine wave) is injected in the biasing current of $1 \mathrm{~mA}$. This causes a DC-shift due to bandwidth limitation of the circuit [12]. The rectification analysis of the circuit classifies the current-mirror transistors M5 and M6 as EMI soft devices. Large operating-point shifts are observed because of asymmetric rectification effects. These transistors will be implemented by their full BSIM3 model, otherwise this effect would not have been modeled. M1-M4 are simplified with the macromodel of Fig.1.

The model contains $\sim 50$ DC-operating-points, sampled along the input signal range. The output load varies in 4 


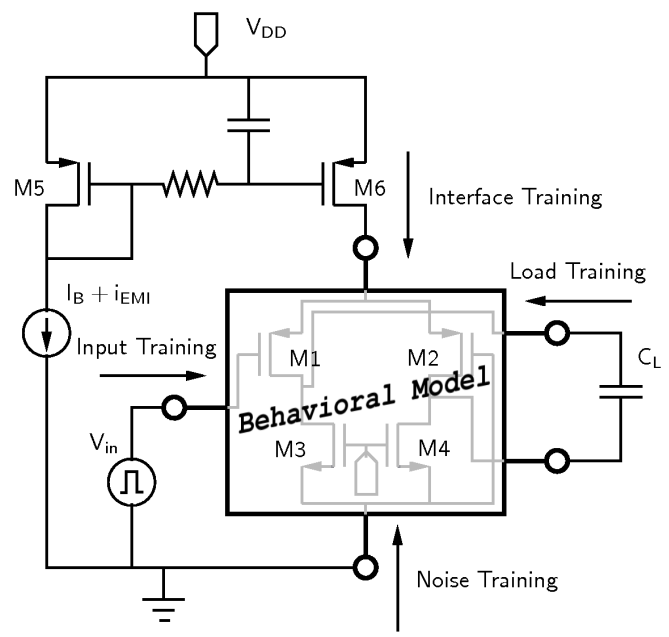

Fig. 3. Circuit schematic of the single-stage opamp with a low-passfiltered current mirror. Transistors M5 and M6 implement their full model for capturing high nonlinearities, due to EMC rectifying effects. The behavioral model is trained with the indicated inputs.

steps from $100 \mathrm{fF}$ to $1 \mathrm{pF}$. The interface between the behavioral model and the full model implementation is trained with $\sim 20$ points, corresponding the DC-shift of M6. This shift was measured with the transient simulations of the original circuit. In 26 minutes, a total of $\sim 4600$ samples were generated on a $2.7 \mathrm{GHz}$ Pentium machine with $3 \mathrm{~GB}$ of RAM.

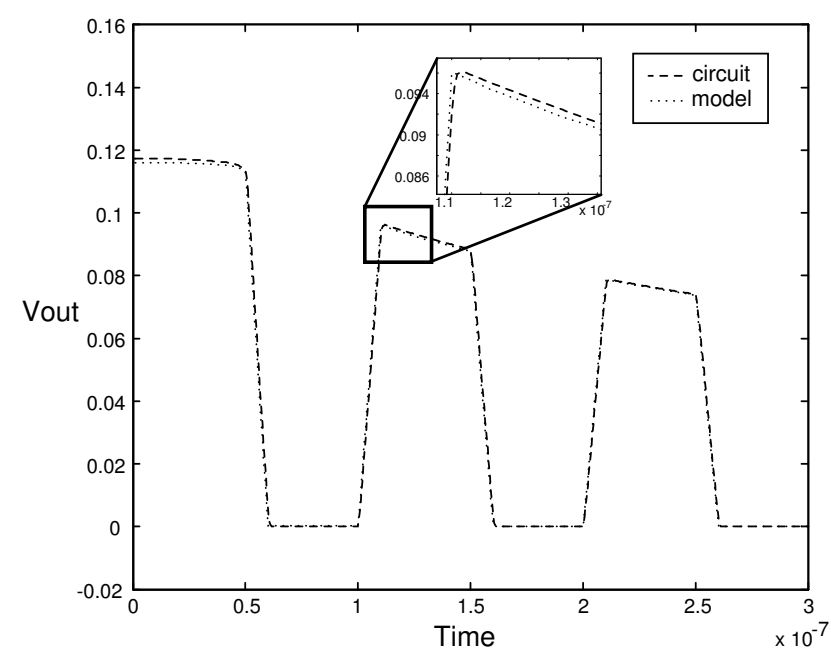

Fig. 4. Output waveform comparison for the opamp output. The gain is gradually pinched off because of the injected EMI noise $i_{E M I}$. The model is almost indistinguishable from the original circuit.

A transient simulation is performed with a pulse-shaped input signal and the biasing current is modulated with the described EMI signal. The resulting output waveforms of the model and the original circuit are plotted in Fig. 4. The 2 waveforms show a very close agreement with an average error of $1.2 \%$ and a maximal error of $\sim 5 \%$, which is comparable with [8] [9]. The obtained accuracy is proportional to the number of samples and shows a high dependency on the skewness of the weighting functions. A main bottleneck of trajectory-based modeling remains the definition, tuning and evaluation of the weighting functions. The DC-operating-point shift is perfectly captured as there is an obvious biasing current pinch-off. To the best of our knowledge, this is the first modeling of rectification effects in behavior models.

Because of the small size of the circuit and the high accuracy, only a modest speedup of $\sim 2.2 X$ is observed. The simulation speedup is expected to increase as the circuit size scales up. A rather small circuit is selected in this paper to demonstrate the concept of layered abstraction modeling and its relevance for EMC purposes.

\section{CONCLUSION}

In this paper we have presented a general approach towards the trajectory-based modeling methodology that allows a flexible implementation of abstraction levels. This algorithm proves to be very accurate in modeling of nonlinearities like rectification effects due to EMI susceptibility. The model has been implemented in a combined VHDL-AMS and SPICE structure and can be simulated with a commercial simulator like ELDO, without any hacks in the simulator engine. A speedup of $2.2 \mathrm{X}$ is realized for a very small circuit, showing a perfect EMI-induced DC-shift modeling. Speedups are expected to be larger for larger circuits. We believe that this method enables a widespread use of trajectory-based modeling for realistic applications that need a reliable anonymization of their intellectual property.

\section{ACKNOWLEDGMENT}

The authors acknowledge the financial support of IWT/MEDEA+ and ON Semiconductor.

\section{REFERENCES}

[1] R. Rutenbar, G. Gielen, and J. Roychowdhury, "Hierarchical modeling, optimization, and synthesis for system-level analog and RF designs," Proceedings of the IEEE, vol. 95, no. 3, pp. 640-669, 2007.

[2] J. Phillips, "Automated extraction of nonlinear circuit macromodels," in CICC, 2000. Proceedings of the IEEE 2000, 2000, pp. 451-454.

[3] R. Freund, "Krylov-subspace methods for reduced-order modeling in circuit simulation," Journal of Computational and Applied Mathematics, vol. 123, no. 1-2, pp. 395-421, 2000.

[4] L. Pillage and R. Rohrer, "Asymptotic waveform evaluation for timing analysis," IEEE Tr. on CAD, vol. 9, no. 4, pp. 352-366, 1990.

[5] K. Gallivan et al., "Asymptotic waveform evaluation via a restarted Lanczos method," Appl. Math. Lett, vol. 7, pp. 75-80, 1994.

[6] J. Phillips and L. Silveira, "Poor man's TBR: a simple model reduction scheme," IEEE Tr. on CAD, vol. 24, no. 1, pp. 43-55, 2005.

[7] M. Rewienski, "A trajectory piecewise-linear approach to model order reduction of nonlinear dynamical systems," Ph.D. dissertation, Massachusetts Institute of Technology, 2003.

[8] S. Tiwary and R. Rutenbar, "Scalable trajectory methods for on-demand analog macromodel extraction," in IEEE DAC, 2005.

[9] N. Dong, J. Roychowdhury, T. Inc, and T. Dallas, "General-Purpose Nonlinear Model-Order Reduction Using Piecewise-Polynomial Representations," IEEE Tr. on CAD, vol. 27, no. 2, pp. 249-264, 2008.

[10] J. Whalen et al., "Computer-Aided Analysis of RFI Effects in Digital IC's," IEEE Tr. on EMC, vol. 21, no. 4, pp. 291-297, Nov. 1979.

[11] J. Loeckx and G. Gielen, "Efficient identification of major contributions to EMI-induced rectification effects in analog automotive circuits," in EMC-Zurich 2006, pp. 148-151.

[12] J. Redoute and M. Steyaert, "Current mirror structure insensitive to conducted EMI," Electronics Letters, vol. 41, no. 21, pp. 1145-1146, 2005 . 